\title{
The Development and Evaluation of a Network for Producing and Sharing
}

\section{Video Presentations}

\author{
Prof. Alaa Sadik \\ South Valley University, Egypt \\ alaasadik@svu.edu.eg
}

\begin{abstract}
This paper describes the technology and methodology used in the development and evaluation of an online network to help instructors produce and share video presentations in a new and innovative way. The network offers an application and platform for recording and sharing video presentations. The application allows instructors to narrate and annotate PowerPoint slides and synchronize a variety of visual aids, including webcam footage, whiteboard, drawing board, and web browser. The platform uses YouTube API to upload and integrate videos with scrollable slide thumbnails. Quantitative and qualitative techniques were implemented to collect data and report on the usability of the network and effectiveness of video presentations. The results of usability evaluation showed that the network was perceived by instructors and students as effective, efficient, and relevance for self- production and sharing of video presentations. In addition, video presentations produced by participants met the criteria of quality of video presentations.
\end{abstract}

Keywords: video, video presentations, video sharing, usability evaluation, e-learning 


\section{Background}

Presentation tools are increasing in popularity and providing powerful tools for creation of instructional materials and accessible information in audio-visual formats. When used appropriately, these tools can support and extend traditional presentations in valuable ways. In lecture settings, Microsoft PowerPoint, for example, has become the dominant presentation tool because it is both readily available and easy-to-use by instructors (Grabe \& Grabe, 2007). It allows instructors to create and manipulate presentations in a wide variety of contexts that can enhance students' interests and engagement (Mills \& Roblyer, 2006). Presentation tools help instructors clearly identify the main points of a topic or activity while still providing the details through presentation (Loisel \& Galer, 2004). Instructors can incorporate multiple types of media formats (e.g., diagram, photo, drawing, sound, animation, video, etc.) that cannot be easily integrated together into one single medium. Learners are also attracted to presentation applications because of graphical, transactional, aesthetic and interactive features they provide. Parette, Blum, Boeckmann \& Watts (2009) suggested that regardless of such concerns and problems related to the use of presentation tools, like PowerPoint, it is no longer an issue of whether to use them or not. Instead, instructors must focus on how they can successfully use them inside and outside classrooms to support learners (Parette et al., 2009).

When deciding how to support the learners, the literature emphasizes the importance of considering the potential possibilities that a video presents (Cunningham \& Friedman, 2009). Proponents of videos argue that there is increasing interest in providing learners with video materials which is demonstrated to be an expanding channel for presentation tools (Sturmey, 2003). Providing video to students is used to support facet-to-face, online, or blended learning. Students can choose when and where to use the material and can spend as long or as little time on each learning activity (Whatley \& Ahmad, 2007). Video is socially acceptable and widely used and supported by multimedia mobile devices and portable media players, and therefore it can be a powerful link between the instructor and students. Also, according to Schwartz \& 
Hartman (2007), video does not have to be stand-alone, like a television program because learners can play, rewind, forward, and pause the video to address their specific needs. It can be used in many ways to encourage interactions between students and the instructors and create engagement.

Martin (1990) found that watching video is considered as a basis for mental activity, because learners already have considerable practice with it in non-educational settings. Research (Zue \& Bergom, 2010; Dey, Burn, \& Gerdes, 2009; Fernandez, Simon, \& Salan, 2009) described many advantages of using video presentations in universities. For example, video presentations allow students to review material at their own pace and location, useful for international students, provides an opportunity to re-organize teaching time, and useful for "equation heavy" disciplines. Recording lectures in video format allows students to catch up if they miss a faceto-face lecture. Video presentations also enable instructors and students to adopt more flexible learning patterns if they wish.

Review of multimedia literature revealed that overcoming the limits of learner working memory requires presenting part of the information being taught in a visual mode and part of it in a verbal mode (Homer, Plass, \& Blake, 2008; Mayer, 2001). Presenting lesson information in both visual and verbal formats helps learners to construct their own knowledge and retrieve information more easily. According to Mayer and Anderson (Mayer \& Anderson, 1991), video can enable improved communication of learning material can aid the retention of verbal information. Mayer (2001) provided a practical set of research-based principles that can help reduce cognitive load in PowerPoint-based video materials. For example, he argued that learners understand a multimedia explanation better when the words are presented as narration rather than on-screen text.

A second finding emphasized the concept of video presence and personalized narration within multimedia environments. Research indicated that although displaying the video of the instructor along with the slides creates a visual distraction, taking audience's attention away 
from the visual information in the slides, presence of the instructor view is important to give the learner a sense of interaction with the instructor (sense of social presence) while watching the video lesson and may improve understanding, even though it adds to cognitive load (Homer, Plass, \& Blake, 2008; Mayer \& Moreno, 1998). Gunawardena (1995) found that social presence is necessary to support participants in technology-based learning environments.

\section{The Problem}

At Sultan Qaboos University (SQU), Sultanate of Oman, faculty members face the need to support student learning by providing new means for presenting curriculum materials, illustrate concepts that are less easily explained through traditional media, support new types of learning opportunities, and provide enrichment activities for students in traditional face-to-face lectures or through e-learning environments. However, many barriers are facing the selection and use of quality learning materials that could be avoided through systems that easily encourage instructors to produce and share course materials themselves. In face-to-face settings, multimedia presentation applications have become the dominant tools because it is both readily available and easy-to-use by instructors. SQU students are also attracted to presentations applications because of graphical, aesthetic and interactive features it provides. However, although PowerPoint presentations are shared with students in a daily basis via e-mail and online slide sharing applications, PowerPoint slides are designed to be delivered by instructors in lectures and need to be paired with the use of LCD projectors and large screens. Usually a presenter should guide the audience through the slides, explain the content to them, keep their interests, and attract their attentions. In other words, slide content and visual features are not substitute for guidance an instructor should deliver. If the learners are not seeing and listening to the instructor, then learning from the slides in isolation will be less valuable or difficult for learners. At the same time, although video hosting solutions, like YouTube provide great solutions for hosting and sharing video content with learners, they do not provide instructors with tools for producing quality video content based on classroom presentations. 


\section{Purpose of the Study}

This study aimed to:

1. Design and develop an online network to help faculty members produce and share quality video presentations.

2. Evaluate the usability of the video presentations network in terms of effectiveness, efficiency and relevance as perceived by instructors.

3. Investigate students' perceived usefulness of online video presentations.

\section{Research Questions}

This study seeks to answer the following questions:

1. How usable is the network as a system for producing and sharing video presentations as perceived by instructors?

2. What is the quality of video presentations produced and shared by instructors?

3. What are students' perspectives about PresentationTube video presentations?

\section{Significance of the Study}

Like many other universities around the world, SQU, has seized on technology as a way to better prepare students and help them in achieving learning objectives. A special center within SQU, called the Center of Educational Technology (CET), was formed to coordinate the SQU's efforts to infuse technology in daily teaching and learning practices. According to CET, infusion of technology like PresentationTube network implies development in improving the performance of students and increasing the capacities of technology use in the university. This study may help CET, faculty members, and students by providing a new simple and realistic way for recording and sharing video presentations that may result is a shift in technology utilization. 


\section{Method}

\section{Design and Development of the Network}

Motivation. The review of the literature and existing classroom technologies above revealed that choosing an appropriate system for recording video presentations by individuals is not easy. There is a wide range of what is known as "conversion", "presentation recording", "screen recording", or "lecture capture" technologies available and used today. Whatley \& Ahmad (2007) distinguished between two different approaches for producing video presentations from classroom presentations: the first is recording a live face-to-face lecture and make it available without editing the original footage. However, when using video of a lecture, much of the interactivity in the form of students' questions and comments should be cut out, because of the low quality of sound and light. The second is recording a "summary presentation" of the lecture in office. The lectures recorded in office are found superior to those recorded in the lecture for a number of reasons: the material could be rehearsed; sound and lighting levels are more controllable; less extraneous noise; unnecessary movements are avoided; and the quality of sound is better (Whatley \& Ahmad, 2007).

The technology used in either approaches range from very simple converter (convert PowerPoint presentation to standard video or Flash video) to highly sophisticated capture stations with multiple cameras and dedicated computers. The majority of these solutions are sophisticated applications designed for campus settings and intended for large-scale distribution. None of these solutions (e.g., Camtasia Studio, authorPoint, Wimba, etc.) has been developed specifically with individual instructors' needs in mind. In addition all these solutions aim to capture lectures and store outputs on a temporary file server to be further processed by technicians, who perform daily checks and upload videos to YouTube. Therefore, the majority of individual instructors will not be able to integrate any of these technologies into their classroom practices. 
Media capture and acquisition application. The main intention of the design phase was to design and build the appropriate capture technique that acquires and synchronizes visual aids simultaneously. In conventional classroom setting, the instructor uses a large wall screen, whiteboard or flipchart and wants to video everything, including him/herself. But if the camera is pointed at screen or play area, the learners would not be able to read from the video because the low quality of the video output (e.g., contrast, reflective surfaces, glare, shadows, small text, limited area, positioning, etc.). This necessitates having a camera operator to pan and zoom as the instructor works. Therefore, the need was emphasized to develop a desktop tool to assist instructors automate the process of synchronizing and recording video presentations. Microsoft Visual Basic, a high level and object-oriented graphical user interface-driven development platform for Windows, was used to design user-interfaces and to code the video presentation recorder. The primary output of this phase was a complete code, a fully functional beta version (prototype) of the video presentation recorder (called PresentationTube Recorder).

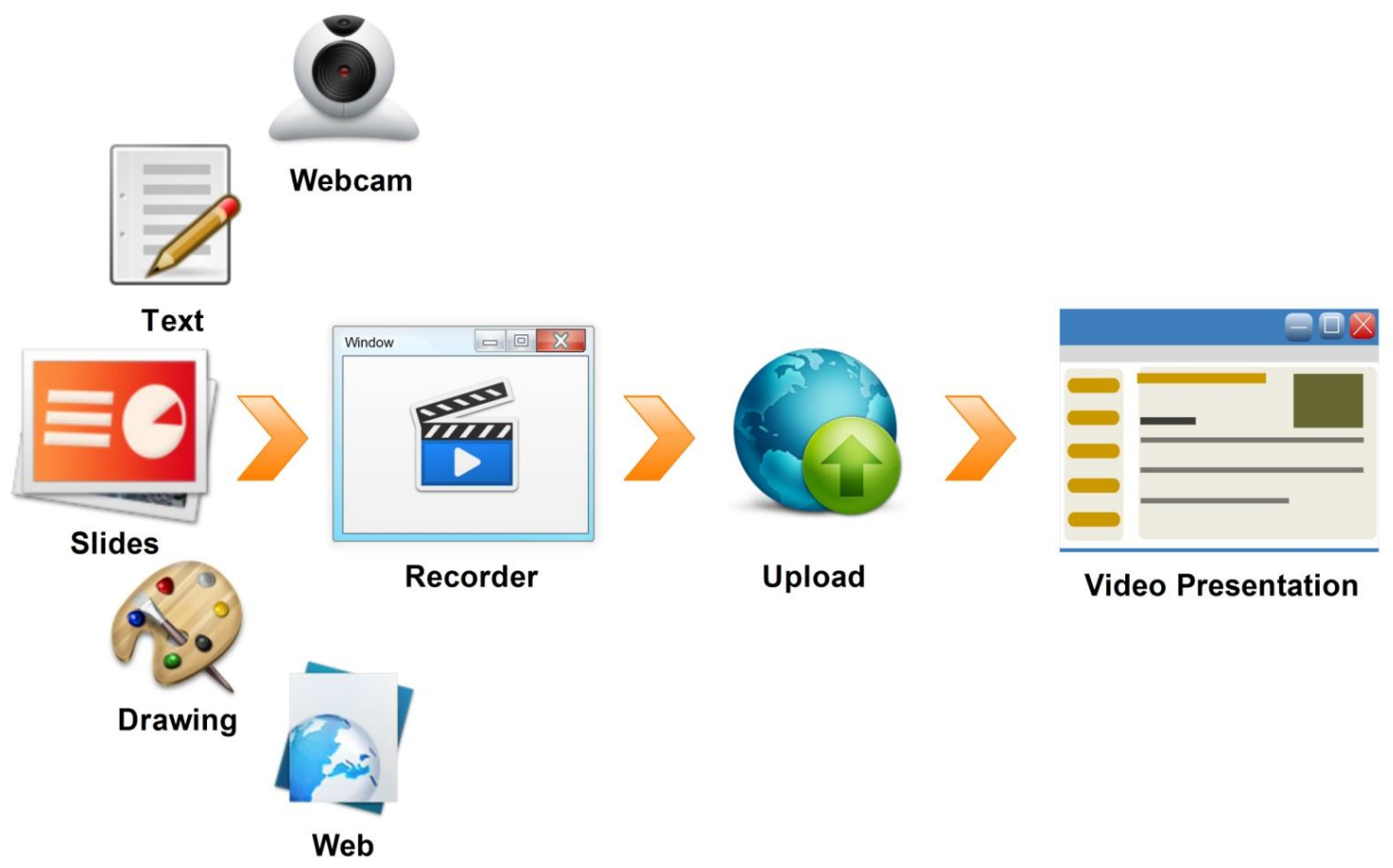

Figure 1 The design of the video presentation recorder 
The recorder has two major aspects - display and capture. The technique involves displaying content in a small window within the software window (see Figure 1). The window allows the instructor to display, manipulate and narrate the content the same way as opened and treated in the full-screen mode. The instructor can navigate forward and back through slides, and employ other features, including slide transitions, text and graphic animation, slide timing, mouse movement, audio effects, embedded video, and pen annotation. The same window is used as a camera viewer to show the presenter's video footage using integrated or external webcam. More than one camera might be connected to give views of the scene from time to time. The instructor can generate professional-looking video presentations by switching between multiple camera views, which is important for creating attractive video materials. The instructor can merge and display the camera video footage inside the content window (picture-in-picture) or add the video footage beside the content window (side-by-side).
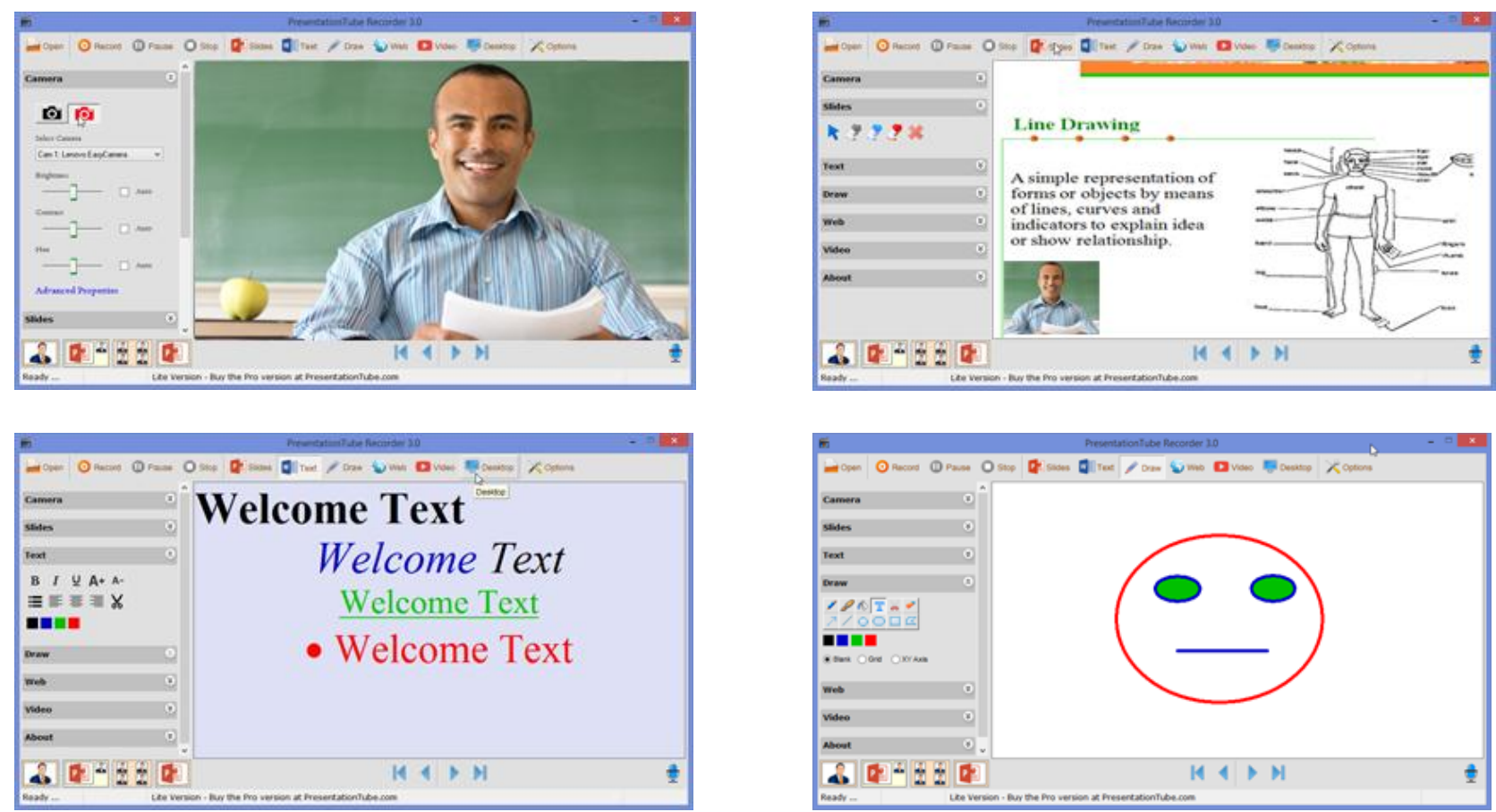

Figure 2 Video presentation recorder - user interfaces

With visual aids, like the drawing board, presenters can draw lines, curves, graphs, and shapes on the screen to emphasize or clarify their ideas, so the demonstration can be clearer. The whiteboard helps instructors to type text while presenting using the keyboard making it an ideal 
tool to add more details, or explain equations using words, numbers, and symbols. This display window allows the instructor to switch between slides, whiteboard, drawing board, and webcam while recording, which simplify the capture process and avoid the problems of managing different visual aids and media. The final outputs of the video presentation recorder are a video file in WMV/AVI format and presentation data file in ZIP format. Both files will be uploaded to PresentationTube platform to create the video and presentation slide thumbnails, as shown later.

To determine whether the prototype met the needs and expectations of instructors and collect user-performance and satisfaction data, the rapid applications development model (RAD) offered a framework within which the software was designed, developed and tested on time and within budget (Scacchi, 2001; Rushby, 1997). The RAD model allowed the developer to rapidly construct early or primitive versions of the recorder that users can evaluate. A series of user-based usability testing tryouts were conducted using one-to-one and small groups of academicians $(n=16)$ at SQU. The computer experience of the volunteers varied. During tryouts, the researcher noticed some user-interface interaction problems and collected useful suggestions from the participants.

The Online Platform. Typically, an educational institute stores the videos on local servers and students can connect to have them streamed to their browsers whenever they want. It is also possible to use a free or paid video streaming network, such as YouTube, Vimeo, WISTIA, Ustream, or DailyMotion to host and stream videos for students to use at any time. Unlike free video streaming services, like YouTube, paid video streaming services provide professional video hosting and streaming solutions, particularly for business and large companies. Some of these services (like YouTube and Vimeo) allow media developers to upload videos directly from their websites using advanced programming techniques called Application Programming Interface (API) and charge users per video file size and streaming bandwidth which is very costly for educational and non-profit purposes. 
YouTube allows users to create their own video channels and upload, stream and embed videos in their own websites for free. In addition, YouTube uses its own advanced API, allowing PresentationTube project to upload videos directly from the website and without the need to have a separate account for each user. For example, an instructor can sign-in to PresentationTube platform and upload a video presentation. The video will be uploaded directly from PresentationTube servers to YouTube media servers for encoding and optimization, and then for embedding in PresentationTube platform. These features allowed YouTube to be the optimal solution for uploading, hosting and streaming video presentations in this project. 
International Journal of Internet Education December, 2016 http://ijie.journals.ekb.eg/ ISSN: $1687-6482$ ivesentation Tube

Record Upload Browse Sign in

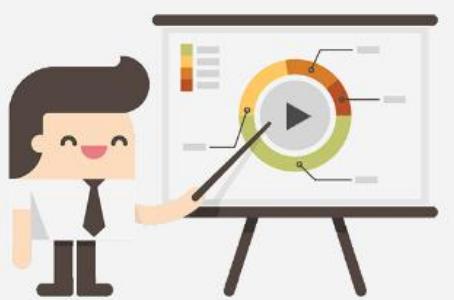

\section{A Network of Video Presentations}

PresentationTube offers a presentation recording software \& online platform to help teachers, students and all business professionals record \& share quality video tutorials!

\section{About Us Sign UD Download Ver. 3.13.1}

$£$ Record The presentation recorder allows you to
narrate and annotate PowerPoint slides,
and synchronize a variety of visual media,
including webcam, whiteboard, drawing
board, Web browser, YouTube video, and screen activity.

\section{U. Upload}

The online platform uses the latest YouTube APIs technology and modern video uploader with progress bar to upload, process, and host the video presentation and combine it with scrollable slide navigator.

$$
\begin{aligned}
& \text { Share } \\
& \text { You can control the privacy level, share } \\
& \text { video presentation link or embed the } \\
& \text { video in any website or blog. The } \\
& \text { audience can move to the respective video } \\
& \text { content and control both the progress and } \\
& \text { length of video presentation. }
\end{aligned}
$$

Featured Presenters
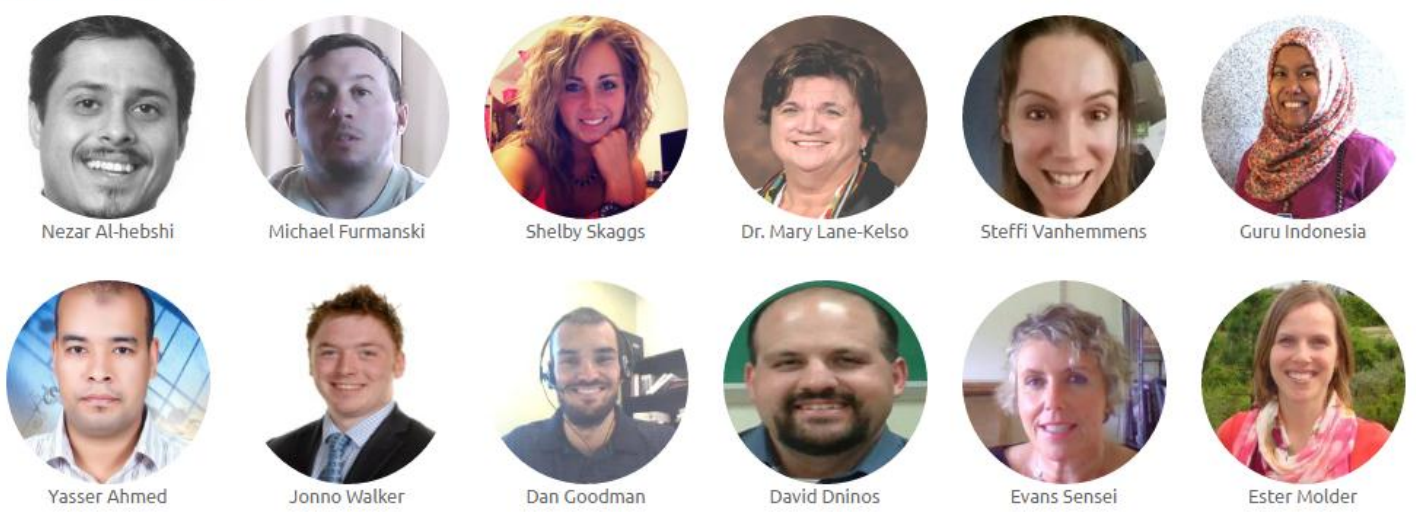

New Uploads
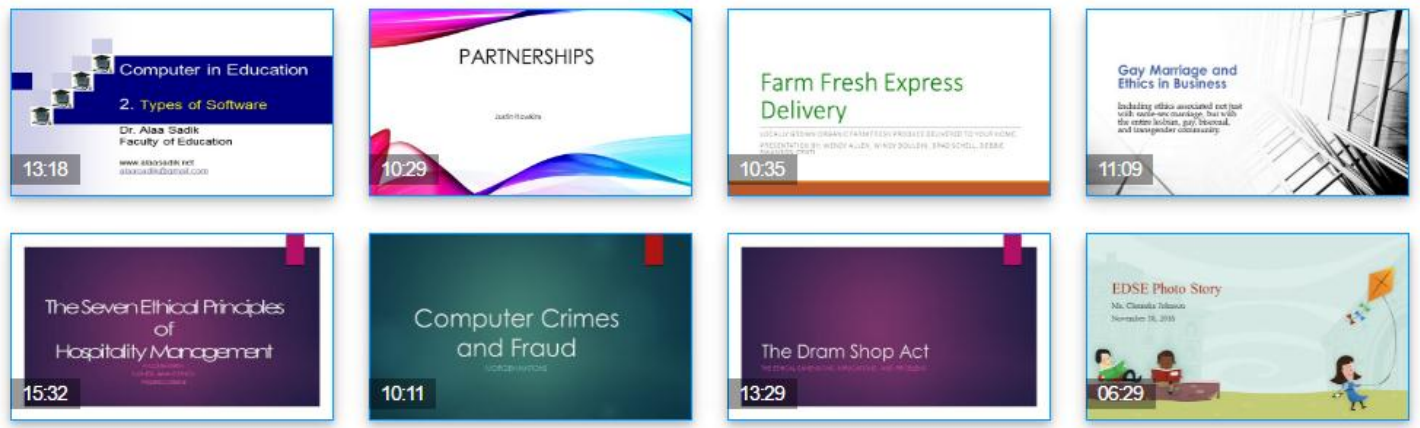

Figure 3 PresentationTube Network- online platform

In contrast to books, which can be consumed at the learner's own pace (much time or less time) and arrangement (any chapter), YouTube video player is designed to be watched at a prescribed rate which means one minute of video content consumed per one minute of watching time in a prescribed direction. This prevents learners from learning at their own pace and their own arrangement, since the video content must be watched in the order that it was created. Although 
YouTube video player allows learners to easily skip forward and back from point to point on the video streamline to a relevant content, the video player does not integrate the video content with the PowerPoint slides. Therefore, PresentationTube platform offered a new way to combine the video content with the presentation slide thumbnails.

Thumbnails are miniature versions of PowerPoint presentation slides similar to slide sorter view in PowerPoint. Thumbnails are arranged in vertical view to allow the learner to browse and navigate through the video content and control both the player and sequence of video presentation. In addition, this association helps to break the forced pace and linear consumption of video content and allows the learner to move to the respective video content and control both the time and progress of video presentation. So, for example, if the learner needs to watch the instructor's explanations on slide 5 , he/she clicks on slide 5 thumbnail to skip directly to the video part associated with this slide. Video presentations are augmented by a discussion forum to enable some form of asynchronous interaction with the presenter to elaborate on issues that might arise from the material presented in the video or respond to students' questions (Whatley \& Ahmad, 2007). PresentationTube network has been made available online for public at www.presentationtube.com in January 2013.

\section{Participants}

Thirty-seven faculty members from College of Education, College of Arts and College of Medicine at Sultan Qaboos University agreed to participate in the project after two e-mail invitations. Many criteria were used for the selection of participants such as self-motivation to participate in the project, diversity of specialization, and technical experience. Of the 37 participants, $31(84 \%)$ instructors attended a series of orientations, seminars, and training workshops and were committed to participate in the project implementation.

\section{Instruments}

The quality of video presentation rubric. The purpose of this evaluation is to assess the video presentations produced by faculty members to explore the extent to which they produced 
quality video presentations for their students using PresentationTube Recorder. Reviewing the literature shows that quality assessment instruments are found appropriate to evaluate multimedia content and video presentations. One such instrument is the scoring rubric. Therefore, the need is emphasized to construct and validate a scoring rubric to evaluate the quality of participants' completed video presentations using indicators in several categories. Based on the objectives of this study, Moskal's six traits and Mertler's framework, the pedagogical and technical attributes of video presentations and their influence on student learning are revealed. These measures are 1) learning objectives; 2) motivation; 3) accuracy of content; 4) sequencing of information; 5) design for learning; 6) text and formatting; 7) quality of audio; 8) quality of graphics; 9) camera positions; 10) transitions and effects; 11) metadata and interoperability; 12) interaction with students; and 13) re-usability of presentation.

In order to ensure that the proposed rubric is appropriate to the desired purpose, constructrelated evidence is examined. In order to gather validity evidence, the accuracy and suitability of the rubric attributes are examined. A panel of five reviewers was asked to review the proposed attributes of evaluation and report on attributes and their characteristics. This review led to fine tuning of the descriptors for the attributes in the rubric. The final attributes and their characteristics are re-constructed and represented in thirteen-attribute scoring rubric with a five point scale: Poor=0; Low=1; Moderate=2; High=3; and Exceptional=4 for greater discrimination within the scale.

PresentationTube usability questionnaire. A usability evaluation questionnaire was developed by the researcher in several phases using both quantitative and qualitative methods. The questionnaire development process occurred in four stages: delineation of relevant domains for the constructs of interest; questionnaire assembly and pilot testing; large-scale field-testing; and validation of instrument scores using factor analytic and correlation methods. The first step of instrument development involved reviewing the literature on software usability evaluation to conceptualize the domains that would directly influence usability of 
PresentationTube network, including the video recorder and the online platform. The review revealed many aspects that fell within three measures: usefulness (effectiveness), ease of use (efficiency), and appeal (satisfaction) (Lohr, Javeri, Mahoney, Gall, Li, \& Strongin, D., 2003). The purpose of the second phase was to use the information in phase one to develop a multidimensional rating scale that could be used to assess the usability of PresentationTube and to assess the content validity of its dimensions. Based on the conceptual definitions of the above measures of usability, each measure was examined for comprehensiveness.

In the third phase, the usability questionnaire with 41 items was field tested with a sample of 7 SQU instructors. An invitation email message with information about the study and link to the online questionnaire was sent to a random sample of instructors at SQU $(\mathrm{N}=75)$ asking them to download and try PresentationTube Recorder, review PresentationTube website then complete the online questionnaire (October 2013). To maximize return rates, the Assistant Dean for Training and Community Services at the College of Education sent an email message requesting cooperation with the researcher. The response rate was monitored over a two-week period. After one follow-up email, responses were received from 32 instructors $(42.5 \%)$.

In the last phase, the psychometric characteristics of the questionnaire were investigated through the use of exploratory factor analyses and Cronbach's alpha. Because the questionnaire was divided into logically different sub-scales, common factor analysis was applied to verify whether the questionnaire measured only one dimension. The inter-correlations show that, overall, each construct was significantly correlated with the other two constructs and with the entire scale. According to Harrison, Seeman and Behm (1991), this result provides further evidence for the consistency of the entire scale and for the convergent validity of each subscale. Therefore, it can be concluded that the three sub-scales and their constructs measure the usability of PresentationTube in a coherent way. The final version of the questionnaire was made available online for instructors to complete at the end of the Fall 2013 semester. 
Interview. Interview was used to clarify and validate usability questionnaire results, where the researcher conducted interview individually with participants. The interview has been scheduled to be conducted after getting questionnaire results from participants. Questions probing positive and negative experiences with the usefulness, ease of use and relevance of PresentationTube were included to obtain any further advantages, limitations, problems, or suggestions from the participants on each section. Interview questions include the following:

1. How was your experience with the Recorder when you used it for first time?

2. What are the most/least useful features you found in PresentationTube Recorder?

3. What are the features you most liked in PresentationTube Recorder?

4. What features/functions would you like to see added to PresentationTube recorder?

5. To what extent PresentationTube network can be of any use in your course?

6. In your opinion what factors make PresentationTube platform superior to other slide sharing or video sharing networks like YouTube?

Students' perspectives to video presentations questionnaire. As a principal aim of this study was to investigate student learning benefits experienced when using PresentationTube video presentations as a learning resource, a questionnaire to solicit students' perspectives about the different aspects of learning benefits of PresentationTube was developed. Since a numerous number of studies (e.g., Park \& Son, 2009; Laird \& Kuh , 2005; Wilson, Wallin \& Reiser, 2003) have developed instruments to measure students' perspectives about learning resources and systems, such as live conferencing, blogs, e-libraries, etc., therefore, various potential indicators were used measure students' perspectives about PresentationTube video presentations. The questionnaire consisted of two sections: the student demographic information section and the rating scale section.

The demographic information section included questions about the frequency of watching video presentations, the location from which they usually access PresentationTube, and the devices they use to watch PresentationTube video presentations. The second section included 
measures to assess students' perspectives. These measures are motivation, satisfaction, usefulness, and effectiveness. All items used a five-point Likert-type scale of potential responses: strongly agree, agree, neutral, disagree, and strongly disagree. The questionnaire was field-tested by a random sample of 35 students at the College of Education. Minor changes to the order and wording of the items resulted from the pre-testers opinions.

The reliability of the questionnaire was computed using coefficient alpha. The reliability of measures shows a high level of internal consistency. A measure of the concurrent validity of the scale is obtained by Pearson's product-moment correlation. Inter-correlation coefficients are found significant at the 0.05 level. The instrument was made available online and instructors were asked to invite their students to respond to the questionnaire two weeks before the end of the Fall 2013 semester.

\section{Implementation}

The purpose of the implementation was to supply information on how the PresentationTube network functions in reality and its effectiveness in achieving the objectives of the project. A three-stage implementation methodology was adopted in this study. The first stage was a series of orientation seminars and training workshops to identify instructors' needs and implementation requirements. The second stage was implementing the project in reality. The third stage consolidated participants' perceptions and examined their online activities throughout the network. At the beginning of the project implementation (started Fall 2013), participants attended five orientation seminar and training workshops organized by the SQU's Center for Educational Technology and Assistant Dean for Training and community Services' Office at the College of Education. Topics included producing and sharing video presentations, the applicability of online video presentations to current teaching contexts at SQU, and their advantages were provided. In addition, more detailed information about using PresentationTube recorder and network was offered via the network blog and Facebook group. The workshops 
emphasized the importance of co-operation among the developer and faculty members for the success of the project and benefits for students.

\section{Results}

The results are organized to address the research questions

\section{The usability of the network as perceived by instructors}

Usability questionnaire. As a principal aim of the evaluation is to investigate how effective, efficient and relevant is the design and functionality of PresentationTube network as perceived by participant instructors, an online questionnaire was administered for this purpose. The overall results show that participants found the network, including the video presentation recorder and the online platform, effective in facilitating the process sharing video presentations with students, easy to use in producing quality video presentations (efficient), and relevant for use in the university by instructors and students (satisfactory).

In terms of effectiveness, participants strongly agreed or agreed that PresentationTube recorder is a useful tool for recording presentations form the comfort of home or office $(76 \%)$, has all the functions to synchronize instructor's video footage and slide content $(81 \%)$, and allows to narrate slide content and annotate slides in a very effective way while presenting (85\%). The majority of participants strongly agreed that $(85 \%)$ that the visual aids included in the recorder, such as the whiteboard and the drawing board allowed them to emphasize or clarify their ideas, so the presentation can be clearer. In addition, instructors found the online platform very effective in uploading video presentations quickly (77\%), sharing presentations with students (89\%), and responding to students' questions and comments on the lecture $(71 \%)$. However, only 39 per cent of the participants believe that PresentationTube video presentations could be used in different teaching settings and scenarios in a flexible way (Table 1).

Table 1 Participants' responses to the usability questionnaire $(n=27)$ 


\begin{tabular}{|c|c|c|c|c|c|c|c|c|}
\hline $\begin{array}{l}\text { Usability } \\
\text { Aspect }\end{array}$ & Items & \multicolumn{5}{|c|}{ Scale } & Mena & SD \\
\hline \multirow{3}{*}{ Effectiveness } & \multirow{3}{*}{14} & Strongly & Agree & Natural & Disagree & Strongly & & \\
\hline & & Agree & & & & Disagree & & \\
\hline & & 34 & 46 & 0 & 14 & 6 & 3.93 & .6324 \\
\hline \multirow{3}{*}{ Efficiency } & \multirow{3}{*}{15} & Very & Somewhat & Neutral & Not very & Not at all & & \\
\hline & & Easy & Easy & & easy & easy & & \\
\hline & & 29 & 35 & 0 & 21 & 25 & 3.87 & .5432 \\
\hline \multirow{4}{*}{ Satisfaction } & \multirow{4}{*}{7} & Definitely & Probably & Possibly & Probably & Very & & \\
\hline & & & & & Not & Probably & & \\
\hline & & & & & & Not & & \\
\hline & & 45 & 20 & 0 & 9 & 6 & 4.2 & .6128 \\
\hline
\end{tabular}

In terms of efficiency, participants rated presentation recorder and platform as very easy to use or somewhat easy $(80 \%)$. They rated the recorder as very easy to use in recording presentations (86\%), importing and displaying slides (90\%), navigation through slides while presenting (89\%), and adjusting camera and video settings (68\%). However, they rated the recorder as somewhat easy to narrate slide content while recording (65\%), and illustrate slide content and guide learners through the presentations using visual aids like the whiteboard and the drawing board (58\%). They argued that adjusting audio and video quality, and switching between different video presentation layouts (such as single, mixed and side-by-side modes) are not very easy using the recorder. In addition, instructors rated the online platform itself as efficient in uploading video files and embedding and sharing video presentations with students (79\%). In terms of satisfaction, participants indicated that, overall, they are satisfied with PresentationTube network (87\%), and would like to be used by other colleagues in the university $(65 \%)$. The results indicate that they liked the way that PresentationTube recorder uses to capture the presentation (67\%), and combine slides with the video for uploading to the online platform $(76 \%)$. 
Interview results. To learn more about the factors affecting the usability of PresentationTube recorder and platform and validate the quantitative findings above, responses from seven participants were collected using the semi-structured interview schedule after one week of ending the implementation period. The responses to the interview questions are organized, analyzed, and coded to address the three aspects of usability. However, since many responses contained multiple and similar beliefs, the number of codes assigned to each question varied. Responses are categorized according to the three aspects of usability and the type of feedback (general or distinctive), as shown in Table 2.

Table 2 Analysis of interview results $(\mathrm{N}=7)$

\begin{tabular}{|c|c|c|}
\hline $\begin{array}{l}\text { Responses to aspects of } \\
\text { usability }\end{array}$ & General patterns (frequency) & $\begin{array}{c}\text { Distinctive viewpoints } \\
\text { (frequency) }\end{array}$ \\
\hline Effectiveness & $\begin{array}{l}\text { - Useful for instructor (1) } \\
\text { - Useful for students (1) } \\
\text { - Provides useful functions (1) } \\
\text { - Increase productivity (2) } \\
\text { - Helps instructor to illustrate } \\
\text { the content (2) } \\
\text { - Produces quality videos (3) }\end{array}$ & $\begin{array}{l}\text { - Not interactive (1) } \\
\text { - Webcam video small (2) } \\
\text { - Save time (2) } \\
\text { - Attendance rate (1) }\end{array}$ \\
\hline Efficiency & $\begin{array}{l}\text { - Very easy to learn (2) } \\
\text { - Easy to use (2) } \\
\text { - Very simple recording process } \\
\text { (3) } \\
\text { - Very easy to upload (2) } \\
\text { - Very easy to share (3) } \\
\text { - User-friendly (4) }\end{array}$ & $\begin{array}{l}\text { - No previous skills required } \\
\text { (1) } \\
\text { - Use existing PowerPoint (3) } \\
\text { - Interesting (2) } \\
\text { - Instant uploading (1) }\end{array}$ \\
\hline Satisfaction & $\begin{array}{l}\text { - Like the recorder (3) } \\
\text { - The website is well-designed } \\
\text { (3) }\end{array}$ & $\begin{array}{l}\text { - Should be used by all } \\
\text { instructors (2) } \\
\text { - Time consuming (1) }\end{array}$ \\
\hline
\end{tabular}




\section{- Students like video \\ presentations (2) \\ - Recommend it to my \\ colleagues (3)}

The first question evoked responses to the overall experience with PresentationTube recorder when used for the first time. An instructor indicated that "when I used PresentationTube for the first time I was amazed to see that simple software can help me to produce video to my students in minutes". A second instructor expressed that "it was very nice experience to learn how to record my class presentations and work from my office”. Similarly, a third response confirmed the previous believes “...when I used it for first time, I liked its interface and found no difficulty while using it. It was interesting to learn and use".

When participants were asked about the most and least useful features they found in PresentationTube Recorder, they indicated that PresentationTube has almost all the features they need to produce video materials. An instructor indicated that "I liked the feature that you can use your already made slides, so you do not have to create them in the program itself. Also, adding and taking out the instructor's face. I liked that there are the other tools like the browser and whiteboard, but was not able to use it because of time limitation and my browser setting. I loved the way you embedded the presentation in my Moodle course". Stating another point of view, a second instructor liked the software and suggested more features to be added. He explained that "it is very interesting to share class presentations as video with my students, but it should provide some extra features for collaborative work. It should provide real time feature". When participants were asked to suggest more new features or functions to be added to PresentationTube, an instructor suggested that "enhancement for instructor's image on video like maybe add some picture effects to reduce the bad lighting some people have". This idea was justified by the point of view of another instructor who indicated that "the instructor's video footage should be positioned in a better way, not inside the slides, so part of the text is 
hidden". In terms of length or recording, an instructor highlighted that "a longer video recording time creates a very large file size that may take a long time to upload".

In terms of factors that make PresentationTube platform better than other slide or video sharing networks like SlideShare and YouTube, an instructor expressed that "the way that PresentationTube uses to combine video with presentation slides is very brilliant. YouTube is a great platform when you need to share a video with your students, but students cannot point to a particular point in the presentation. In PresentationTube students can follow and control the video presentations". Additionally, another instructor stated that "PresentationTube helped me to make my presentations clearer. It makes presentation look wonderful as interactive video and slides than any other. PresentationTube changed the way I share course materials and information with my students. With just a few clicks, I can open my presentation, add my picture, and narrate the slides".

The quality of video presentations. To assess the extent to which instructors produced quality video presentations using PresentationTube, the Quality of Video Presentation Rubric was used to assess video presentations uploaded and shared via PresentationTube platform. Each video presentation was assessed using the rubric since the project was relatively small and is feasible to examine all the video presentation. The researcher and three research-assistants, who received their bachelor degrees in education, and attended a training workshop in using the rubric, assessed 187 video presentations. To improve consistency between evaluators, a sample of video presentations was assessed for practice and to enhance agreement among evaluators. Scores for each video presentation are analyzed and represented as a set of averaged ratings, one per criterion, and summarized as a final average covering the thirteen criteria used in the rubric. A total number of 186 videos were assessed. Videos varied in length and quality, but most videos were about 15-20 minutes long.

Overall, assessment of video presentations showed that, instructors did well in their recordings and their videos met most of the pedagogical and technical attributes of quality video 
presentations. The majority of video presentations (72\%) were exceptional in terms of learning goals, accuracy, motivation, quality of graphics, and interaction with students via discussion boards. The rubrics scores revealed that video presentations have many features that attract students' attention (such as inclusion of multimedia elements, interactivity, and length) are likely to show an interest in learning with the video presentations. Video presentations also were rated high in terms of design of presentation, text formatting, camera positions, and reusability of the presentation. In addition, most video presentations (86\%) showed clear content narration and effective use of annotations that help students to understand the main points in the presentation. The design of presentations took into account the differences in students' needs, allowing different cases of students to benefit from the videos (Table 3).

Table 3 Quality of video presentations produced by instructors ( $\mathrm{n}=186$ video presentations)

\begin{tabular}{|c|c|c|c|c|c|c|c|c|}
\hline Criteria & $\hat{\theta}$ & ¿ & ${ }_{\xi}^{5} \mathbb{N}$ & 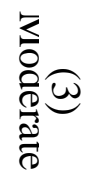 & 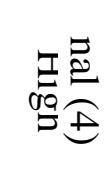 & 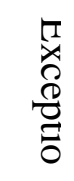 & $\begin{array}{l}\text { Mean of } \\
\text { Rating }\end{array}$ & StdDev \\
\hline 1. Learning objectives & & & & & & $\checkmark$ & 3.32 & .9274 \\
\hline 2. Motivation & & & & & & $\checkmark$ & 3.31 & .6265 \\
\hline 3. Accuracy of content & & & & & & $\checkmark$ & 3.74 & .5956 \\
\hline 4. Sequencing of information & & $\checkmark$ & 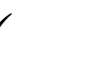 & & & & 1.36 & .5476 \\
\hline 5. Design for learning & & & & & $\gamma$ & & 3.01 & .6434 \\
\hline 6. Text and formatting & & & & & 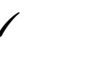 & & 2.51 & .5744 \\
\hline $\begin{array}{l}\text { 7. Audio narration \& } \\
\text { annotations }\end{array}$ & & & & & r & & 1.69 & .4977 \\
\hline 8. Quality of graphics & & & & & & $\checkmark$ & 3.05 & .8562 \\
\hline 9. Camera positions & & & & & 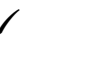 & & 2.86 & .7751 \\
\hline 10. Transitions and effects & & & $\checkmark$ & & & & 2.11 & .6245 \\
\hline 11. Metadata and interoperability & & & $\checkmark$ & & & & 1.91 & .4235 \\
\hline 12. Interaction with students & & & & & & $\checkmark$ & 3.63 & .8380 \\
\hline 13. Re-usability of presentation & & & & & $\gamma$ & & 3.32 & .6491 \\
\hline erage Score & & & & 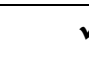 & $\bar{t}$ & & 2.79 & .1645 \\
\hline
\end{tabular}


However, scores revealed that camera positions and interaction with students via eye contact need to be improved by ensuring the instructor positions the camera appropriately. On a practical note eye contact with students when recording the video is to be encouraged, by ensuring the instructor looks into the camera, not slides or computer screen, most of the time. In terms of transitions and effects, quality of audio, and graphics the analysis demonstrated that instructors were not able to make use of the all the technical features of PresentationTube, in order to enhance the presentation of their video presentations (Table 3). The scores show that slide transitions effects are mostly smooth but may be inappropriate or distracting. The biggest challenge issue for instructors was the tight integration between the audio narration and slides movements. In a significant number of video presentations $(26 \%)$ it was found that sound is inconsistent with slides and transition effects when moving from slide to another.

Students' perspectives. By the end of Fall 2013, 218 out of 693 students $(31.5 \%$ of enrolled students) completed the questionnaire. The data from the demographic information section indicated that $91 \%$ of students always or frequently used to watch the video presentations provided by the course instructor. The majority of students used their own laptops $(56 \%)$ or smart phones $(34 \%)$ to watch video presentations from the university network $(69 \%)$ or home (19\%). In terms of students' satisfactions with PresentationTube network, the vast majority of students expressed that they liked the way that PresentationTube uses to combine and present videos with slides $(87 \%)$. In addition, 77 per cent of students indicated that they accessed PresentationTube video presentations without any problem in loading or watching videos (69\%). Students believe that video presentations are effective resources for learning when they missed the lecture (59\%), helped them to understand the topics they could not understand in the lecture $(62 \%)$, and allowed them to interact with the instructor via PresentationTube platform by asking questions and posting comments to the video (68\%).

In terms of usefulness, students found PresentationTube as a useful resource for learning. The majority of students expressed that they did not need to take class notes (70\%) or worry about 
language difficulties in non-Arabic classes (59\%). Overall, students found PresentationTube very encouraging and effective way for learning. Students provided useful comments in the open-ended questions regarding the effectiveness and usefulness of PresentationTube. These comments can be summarized as follow:

1. PresentationTube allowed students to catch up if they miss a lecture. Students who were absent believe that they would able to learn from video presentations the same as students who attended the same lecture in person.

2. Students believe that video presentations improved their learning and increased their overall level of satisfaction and confidence with the course.

3. Video presentations are useful for equation heavy or complex disciplines.

4. Some students rarely ask questions in lectures, particularly in large groups, and prefer to focus on the video presentations.

5. Students are more likely to interact with the instructor after watching video presentations when they have more chances to consider difficult topics carefully.

6. PresentationTube videos provided students with a valuable resource to complement their studies.

7. PresentationTube video player allows students start, stop, rewind, and skip the video content to address their specific needs.

\section{Discussion and Recommendations}

Faculty members have professional and moral responsibilities to provide their students with quality content and resources to support their learning. In this study, the need was emerged to develop and implement an online network to help faculty members at SQU produce and share quality video presentations with their students. To meet these needs, PresentationTube network which offer a video presentation recording application and online video sharing platform has been developed by the author. The recorder allows instructors to narrate and annotate PowerPoint slides and synchronize a variety of visual aids, including webcam video footage, whiteboard, drawing board, and web browser. The platform uses YouTube API to upload 
videos and integrate them with scrollable slide thumbnails, allowing students to move to the respective video content and control both the progress and length of video presentation. The usability questionnaire and individual interviews provided very encouraging feedback regarding the usability of PresentationTube network. Instructors favored the video presentation recorder and found it useful in producing video presentations, easy to use, and satisfactory. More positive responses than negative comments were provided in individual interviews.

Davis (1989) argued that perceived usefulness and ease of use of a system are each highly correlated with self-reported use and future use. The importance of the ease of use and liking of PresentationTube is that it has a significant positive relationship with future adoption of the network in the university. In addition, Rogers (1995) found that the relative advantage (usefulness), as perceived by instructors, is positively related to the innovation's rate of adoption. In other words, faculty members are more likely to use PresentationTube they perceive as easy to use and useful for producing and publishing video presentations for their students.

Analysis of students' questionnaire indicated that they found PresentationTube videos useful for their understanding and revision of lectures. They also found PresentationTube effective on occasions when they had missed a lecture, or needed some help to understand a topic or to help put the material into perspective. In addition, addressing learners using personalized narration allowed them to believe that they are active participants in the video presentations, thereby improving learning and possibly motivation.

However, investigating whether PresentationTube video presentations influenced students' learning or cognitive achievement such as information recall and summarization of main ideas was out of the scope of this study. Therefore, future research can investigate whether PresentationTube actually can improve students' learning and how instructor's personalized narration can influence comprehension of presentations. 
The possibilities for future development and research are exciting. With respect to PresentationTube development, it should be considered a work in progress. Feedback from instructors and students can provided useful guidelines about what and how features need to be improved or added. For example, participants faced some challenges during the implementation period such as creating balance between face-to-face teaching duties and videoing all necessary content within a fixed schedule. Many instructors were not able to shift to more studentcentered classes and how to adopt the new video presentations to meet their students' needs and enhance instructor-student interaction inside and outside classrooms. Therefore, instructors may need more training on how to integrate PresentationTube video presentations successfully to its fullest potentials.

In addition, further research is needed to investigate the design of content, and develop principles for the design of effective video presentations that are consistent with what students need and how they learn from online video presentations. In addition, the role of PresentationTube video presentations in improving student learning and comparisons of how students learn from these videos versus face-to-face classes need more investigation. Lastly, more studies are needed to be directed at determining which learning approaches and environment settings that result in good learning outcomes when used with PresentationTube. 


\section{References}

Allen, D. \& Tanner, K. (2006). Rubrics: tools for making learning goals and evaluation criteria explicit for both teachers and learners. Life Sciences Education, 5(3), 197-203.

Copley, J. (2007). Audio and video podcasts of lectures for campus-based students: Production and evaluation of student use. Innovations in Education and Teaching International, 44(4), 387-399.

Cunningham, A. \& Friedman, A. (2009). Captivating Young Learners and Preparing 21st Century Social Studies Teachers: Increasing Engagement with Digital Video. In I. Gibson et al. (Eds.), Proceedings of Society for Information Technology \& Teacher Education International Conference 2009 (pp. 1797-1803). Chesapeake, VA: AACE.

Davis, F. D. (1986). A technology acceptance model for empirically testing new end-user information systems: Theory and results. Unpublished doctoral dissertation. Cambridge, MA: MIT Sloan School of Management.

Dey, E.L., Burn, H.E., and Gerdes, D. (2009). Bringing the classroom to the Web: Effects of using new technologies to capture and deliver lectures. Research in Higher Education, 50 (4), 377-393.

Echo360 and the Feedback Loop (2010). Capturing Student Perspectives about Lecture Recordings. Retrieved from http://echo360.com/student-survey.

Fernandez, V., Simon, P. and Salan, J. (2009). Podcasting: A new technological tool to facilitate good practice in higher education. Computers and Education, 53, 385-392.

Grabe, M., \& Grabe, C. (2007). Integrating technology for meaningful learning. (5th ed.), New York, NY: Houghton Mifflin Company

Gunawardena, C. (1995). Social presence theory and implications for interaction and collaborative learning in computer conferences. International Journal of Educational Telecommunications, 1, 147-166. 
Harrison, J. Seeman, J. Behm, R. (1991). Development of a distance education assessment instrument. Educational Technology Research \& Development, 39(4), 65-77.

Homer, B., Plass, J., \& Blake, L. (2008). The effects of video on cognitive load and social presence in multimedia-learning. Computers in Human Behavior, 24, 786-797.

Lohr, L., Javeri, M., Mahoney, C., Gall, J., Li, K. \& Strongin, D. (2003). Using Rapid Application Development to Improve the Usability of a Preservice Teacher Technology Course. Educational Technology Research \& Development, 51(2), 41-55.

Loisel, M. \& Galer, R. (2004). Uses of PowerPoint in the 314L Classroom. Computer Writing and Research Lab. White Paper Series: \#040505-3. Retrieved from http://workgroups.cwrl.utexas.edu/main/sites/default/files/040505-3.pdf Martin L. (1990). Detecting and defining science problems: A study of video-mediated lessons. In Moll, L. (Ed.), Vygotsky and education: Instructional implications and applications of sociohistorical psychology, Cambridge (pp 372-402): Cambridge University Press, MA.

Mayer, R. (2001). Multimedia-learning. New York: Cambridge University Press.

Mayer, R. \& Moreno, R. (1998). A split-attention effect in multimedia-learning: evidence for dual processing systems in working memory. Journal of Educational Psychology, 90, 312-320. Mayer, R. \& Anderson, R. (1991). Animations need narrations: An experimental test of a dual coding hypothesis. Journal of Educational Psychology, 83(4), 484-490.

Mertler, C. A., \& Vannatta, R. A. (2002). Advanced and multivariate statistical methods: Practical application and interpretation (2nd ed.). Los Angeles: Pyrczak.

Mills, S. C., \& Roblyer, M. D. (2006). Technology tools for teachers: A Microsoft Office® tutorial (2nd ed.). Upper Saddle River, NJ: Pearson Merrill Prentice Hall.

Moskal, Barbara M. (2003). Recommendations for developing classroom performance assessments and scoring rubrics. Practical Assessment, Research \& Evaluation, 8(14). Retrieved from http://PAREonline.net/getvn.asp?v=8\&n=14 
Nelson Laird, T. F. \& Kuh, G. (2005). Student experiences with information technology and their relationship to other aspects of student engagement. Research in Higher Education, 46(2), 211-233.

Parette, H. P., Blum, C., Boeckmann, N. M., \& Watts, E. H. (2009). Teaching word recognition to young children using Microsoft PowerPoint coupled with direct instruction. Early Childhood Education Journal, 36, 393-401.

Park, C.N. \& Son, J.B. (2009). Implementing Computer-Assisted Language Learning in the EFL classroom: teachers' perceptions and perspectives. International Journal of Pedagogies and Learning, 5(2), 80-101.

Rogers, M. (1995). Diffusion of Innovation, 4th ed., New York: The Free Press.

Rushby, J. (1997). Quality criteria for multimedia. Association for Learning Technology Journal, 5(2), 18-30.

Scacchi, W. (2001). Process Models in Software Engineering. In Marciniak, J. (Ed.), Encyclopedia of Software Engineering, 2nd Edition, New York: John Wiley and Sons, Inc.

Schwartz, L., \& Hartman, K. (2007). It is not television anymore: Designing digital video for learning and assessment. In R. Goldman, R. Pea, B. Barron, \& S. J. Derry (Eds.), Video research in the learning sciences (pp. 349-366). Mahwah, NJ: Lawrence Erlbaum Associates. Whatley, J. and Ahmad, A. (2007). Using Video to Record Summary Lectures to Aid Students' Revision. Interdisciplinary Journal of Knowledge and Learning Objects, 3, 185-196.

Wilson, K. R., Wallin, J. S., \& Reiser, C. (2003). Social stratification and the digital divide. Social Science Computer Review, 21(2), 133-143.

Zue, E. and Bergom, I. (2010). Lecture Capture: A guide for Effective Use. Tomorrow's Professor, Email Newsletter Number 1018. 